\title{
CKDu: the known unknowns
}

\section{A. S. Abeyagunawardena ${ }^{1} \cdot$ R. Shroff ${ }^{2}$}

Received: 30 April 2020 / Accepted: 1 May 2020 / Published online: 15 May 2020

(C) IPNA 2020

Chronic kidney disease of uncertain aetiology $(\mathrm{CKDu})$ is defined as an impairment of kidney function in the absence of a predisposing factor for kidney disease such as diabetes mellitus, chronic hypertension, snake bite with systemic envenomation, chronic glomerulonephritis or obstructive nephropathy [1]. Although commonly seen in Central America, patients with a similar clinical picture have also been reported in Sri Lanka, parts of India and the Balkan region. Meso-American nephropathy $(\mathrm{MeN})$ refers to chronic kidney disease corresponding to a CKDu hotspot in the Pacific Coast lowlands of El Salvador, Nicaragua, Guatemala and Costa Rica [2]. Although first reported in young adult males involved in agriculture, there are emerging reports of CKDu in women and children in rural agricultural communities, challenging the aetiopathogenesis of this condition.

A study from Leibler et al. in this issue of Paediatric Nephrology challenges current theories about the aetiology of $\mathrm{CKDu}$ and demands urgent attention from the international nephrology community to address a global disease in vulnerable, economically disadvantaged people. The authors report a cross-sectional study of 210 youths, aged 7-17 years residing predominantly in a CKDu endemic region of Nicaragua. The authors had noted that CKDu presented at a younger age in Central America and hypothesise that subclinical kidney injury begins prior to working life. Participants were selected from rural regions dominated by agriculture which has a high prevalence and mortality in adults with $\mathrm{MeN}$ and compared with a reference community from an urban capital city of Nicaragua, which recorded a lower adult MeN prevalence. Urinary concentrations of biomarkers of kidney injury such as neutrophil gelatinase-associated lipocalin (uNGAL), kidney injury molecule 1 (uKIM-1), interleukin-18 (uIL-18), monocyte

A. S. Abeyagunawardena asiriabey26@gmail.com

1 Department of Paediatrics, Faculty of Medicine, University of Peradeniya, Peradeniya, Sri Lanka

2 Great Ormond Street Hospital for Children, London, UK chemoattractant protein 1 (MCP-1) and chitinase-3-like protein 1 (YKL-40), as well as urine and serum creatinine and Schwartz estimation of glomerular filtration rate (eGFR), were measured and compared with reference values from healthy children.

Leibler et al. found that median uNGAL, uIL-18 and uKIM-1 concentrations in their study population exceeded healthy reference values, indicating renal injury. A 1-year increase in age was associated with a $40 \%$ increase in the odds of being in the highest quartile of uNGAL. Youth who reported ever experiencing dysuria had 2.5 times the odds of having uNGAL concentrations in the top quartile. Moreover, $9 \%$ of the subjects had a low eGFR $(\leq 100 \mathrm{ml} / \mathrm{min} / 1.73 \mathrm{~m} 2)$, while $29 \%$ showed evidence of hyperfiltration (eGFR $\geq 160 \mathrm{ml} / \mathrm{min} /$ $1.73 \mathrm{~m}^{2}$ ), both indicative of renal dysfunction. All biomarker concentrations were higher in girls compared with boys. It is noteworthy that $19.5 \%$ of youth in this study had urinary biomarker concentrations in the upper quartile for three or more biomarkers evaluated. The authors conclude that children between 7 and 17 years of age who live in a MeNendemic agricultural region are at high risk of subclinical kidney injury prior to occupational exposures. The overall lack of associations between biomarker concentrations and eGFR is concordant with the researchers' hypothesis that change in kidney function represents a relatively late stage event in kidney disease.

\section{Global perspective}

CKDu was first described in Central America in 2002 and in the rural population of the North Central Province of Sri Lanka in 2000 [3, 4]. The disease is often fatal due to rapid disease progression and lack of dialysis or transplant options in these geographic areas. CKDu in affected zones of Central America and Sri Lanka has begun to reach epidemic proportions [1,2], and the high cost involved in the management of end stage kidney failure due to $\mathrm{CKDu}$ has become a national health issue. It is interesting to note that in the cohort described 
by Leibler et al., girls had higher biomarker levels. However, most epidemiological studies describe that predominantly male farmers are affected with symptomatic CKDu [5].

\section{Aetiology of CKDu}

Some community-based studies have estimated that more than 10 years of continual exposure to an environmental toxin are required before the clinical manifestations of CKDu appear [1]. The only published report of children being affected with $\mathrm{CKDu}$ was based on screening with urinary albumin: creatinine ratio (ACR) and serum creatinine [6], and therefore, the validity of these findings are debatable. Leibler et al. present the first report of CKDu in children detected by using a panel of tubular biomarkers, thus supporting the observation of a prolonged subclinical period. However, genetic vulnerability, exposure to greater concentrations of the causative toxin, strenuous manual labour with associated dehydration, protein calorie malnutrition, micronutrient deficiencies and low birth weight may reduce this subclinical period [1].

Despite two decades of intensive research and debate, the specific aetiological agent causing CKDu remains elusive. Data from published literature strongly suggest that $\mathrm{CKDu}$ has a multi-factorial origin, namely, source of drinking water containing nephrotoxic factors, lifestyle changes, farming practices, environmental conditions and recurrent dehydration [7]. Histopathological studies in CKDu patients have revealed tubular interstitial lesions, suggesting a potential toxin involved in the causation of CKDu [8]. A large number of causes related to agriculture have been suggested as aetiological possibilities for CKDu. These include heat/dehydration, infection/inflammation and the possible role of water contamination with heavy metals and/or pesticides. The majority of people living in CKDu-affected areas consume water from domestic stagnant and shallow water wells which do not communicate with the underground water table or deep tube wells. Interestingly, the incidence and prevalence of CKDu is low among people who use surface water from dynamic water sources, such as natural springs, streams, lakes and reservoirs for drinking purposes. However, studies conducted in Sri Lanka and other CKDu-affected countries have failed to provide convincing evidence to prove that the well water is contaminated with causative agents for CKDu such as fertilizers, pesticides or heavy metal $[1,7]$, such that CKDu remains an unknown, yet devastating condition.

\section{Biomarkers and CKDu}

In early 2000 , the detection of urine protein excretion by urine dipstick was used extensively as a screening tool for CKDu. Urine dipstick examination can be carried out in the field with minimum resources and expertise, thus making it a popular screening tool. Since CKDu is a tubular disease and urinary protein excretion is a late development, subjects, in whom the initial dipstick was negative, presented later with advanced stage of disease, disputing the validity of the initial screening process [6]. Hence, the ACR too becomes a non-specific and insensitive test that should not be used for diagnosis or screening. However, biomarkers that are tubular-specific are excreted at an early stage of CKDu and can be used as a sensitive and specific test for screening and early diagnosis as demonstrated by Leibler et al. As yet, tubular biomarkers are seldom being used as a screening tool in affected countries mainly because they are prohibitively expensive [9]. However, if one or two of the five biomarkers used by Leibler et al. can detect the disease early, it may still be cost-effective, as providing renal replacement therapy is an even greater challenge in resource poor countries.

\section{Future directions}

We hope that this study from Leibler et al. and their ongoing work in the field of CKDu will add new knowledge in dealing with this devastating and entirely preventable disease. The fact that children who have never been exposed to manual labour of farming have evidence of renal injury suggests that water contamination may play a role in the initial pathogenesis of the disease. This may provide important scientific evidence to convince policy makers to provide drinking water through the process of reverse osmosis or rain water harvesting systems in CKDu-affected areas. Additionally, biomarkers may be useful for the localization of injury to a specific site in the nephron, as well as for monitoring disease progression and severity $[10,11]$. Future research may establish a single, or small panel of, sensitive and specific biomarker(s) of CKDurelated kidney injury in order to monitor early-life risk factors. Research could be undertaken to understand whether some rural communities are genetically predisposed to renal injury in childhood. Determining such risk factors will help the health sector establish better management strategies to minimize CKDu in affected populations as well as focus on early detection and appropriate interventions at an early stage when kidney injury from CKDu may be halted or even reversed.

\section{References}

1. Wimalawansa SJ (2016) The role of ions, heavy metals, fluoride, and agrochemicals: critical evaluation of potential aetiological factors of chronic kidney disease of multifactorial origin (CKDmfo/ $\mathrm{CKDu}$ ) and recommendations for its eradication. Environ Geochem Health 38:639-678 
2. Ordunez P, Martinez R, Reveiz L, Chapman E, Saenz C, da Silva AS, Becerra F (2014) Chronic kidney disease epidemic in Central America: urgent public health action is needed amid causal uncertainty. PLoS Negl Trop Dis 8(8):e309

3. Trabanino RG, Aguilar R, Silva CR, Mercado MO, Merino RL (2002) End-stage renal disease among patients in a referral hospital in El Salvador. Rev Panam Salud Publica 12(3):202-206

4. Lanerolle RD, Nanayakkara S, Sheriffdeen AH, Sheriff R (2000) Demographic characteristics of end stage renal disease in Sri Lanka. J Ceylon Coll Phy 33:3

5. Almaguer M, Herrera R, Orantes CM (2014) Chronic kidney disease of unknown etiology in agricultural communities. MEDICC Rev 16:09-15

6. Agampodi SB, Amarasinghe GS, Naotunna PGCR, Jayasumana CS, Siribaddana SH (2018) Early renal damage among children living in the region of highest burden of chronic kidney disease of unknown etiology (CKDu) in Sri Lanka. BMC Nephrol 9:115

7. Edirisinghe E, Manthrithilake H, Pitawala H, Dharmagunawardhane HA, Wijayawardane RL (2018) Geochemical and isotopic evidences from groundwater and surface water for understanding of natural contamination in chronic kidney disease of unknown etiology $(\mathrm{CKDu})$ endemic zones in Sri Lanka. Isotopes Environ Health Stud 54:244-261
8. Badurdeen Z, Nanayakkara N, Ratnatunga NV, Wazil AW, Abeysekera TD, Rajakrishna PN, Thinnarachchi JP, Kumarasiri R, Welagedera DD, Rajapaksha N, Alwis AP (2016) Chronic kidney disease of uncertain etiology in Sri Lanka is a possible sequel of interstitial nephritis. Clin Nephrol 86(13):106

9. Bolignano D, Lacquaniti A, Coppolino G, Donato V, Campo S, Fazio MR, Nicocia G, Buemi M (2009) Neutrophil gelatinaseassociated lipocalin (NGAL) and progression of chronic kidney disease. Clin J Am Soc Nephrol 4(2):337-344

10. Bolignano D, Donato V, Coppolino G, Campo S, Buemi A, Lacquaniti A, Buemi M (2009) Neutrophil gelatinase-associated lipocalin (NGAL) as a marker of kidney damage. Am J Kidney Dis 52(3):595-605

11. Ekanayake EMDV, Mohammed Abdul KS, Thakshila WAKG, Jayasinghe SS, Chandana EPS, Jayasumana C, Siribaddana SH, De Silva PMCS (2016) Urinary biomarkers KIM-1 and NGAL may predict chronic kidney disease of uncertain etiology $(\mathrm{CKDu})$ among sugarcane farmers in Sri Lanka. PLoS Negl Trop Dis 10(9): e0004979

Publisher's note Springer Nature remains neutral with regard to jurisdictional claims in published maps and institutional affiliations. 